\title{
Diagnosis and treatment of somatoform disorders
}

\author{
Jonathan K. Smith, MD \\ Ralph F. Józefowicz, MD
}

\section{Summary}

Many patients with somatoform disorders present to the neurology clinic, often after extensive evaluation that has left the patient and multiple other physicians frustrated. Knowledge of the typical characteristics of particular disorders enables the clinician to arrive at a positive diagnosis and facilitate referral to appropriate services. Using a series of representative cases, we review the DSM-IV-TR somatoform disorders most likely to present to the practicing neurologist, highlighting the epidemiologic features, typical presentations, and possible therapeutic approaches to each condition.

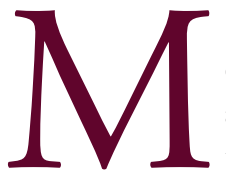

edically unexplained symptoms are common in neurology, constituting anywhere from $20 \%$ to $60 \%$ of outpatient neurology visits. ${ }^{1,2}$ In addition to excluding potential medical causes, the neurologist must also consider somatoform disorders, which the DSM-IV-TR defines as physical symptoms suggestive of a medical condition but unexplained by an underlying disease or mental disorder. ${ }^{3}$ Similar presentations occur in factitious disorder (FD) and malingering, which feature conscious production of symptoms.

The clinician may be frustrated by the patient whose symptoms defy explanation despite exhaustive workup, and this frustration can mount if the patient refuses to agree that all reasonable possibilities have been ruled out. Understanding the presentation of typical somatoform complaints can aid in making a positive diagnosis instead of discharging the patient with the dismissive assertion that there is no evidence for a neurologic disease. To that end, we present cases of common somatoform disorders, along with a brief review of the literature.

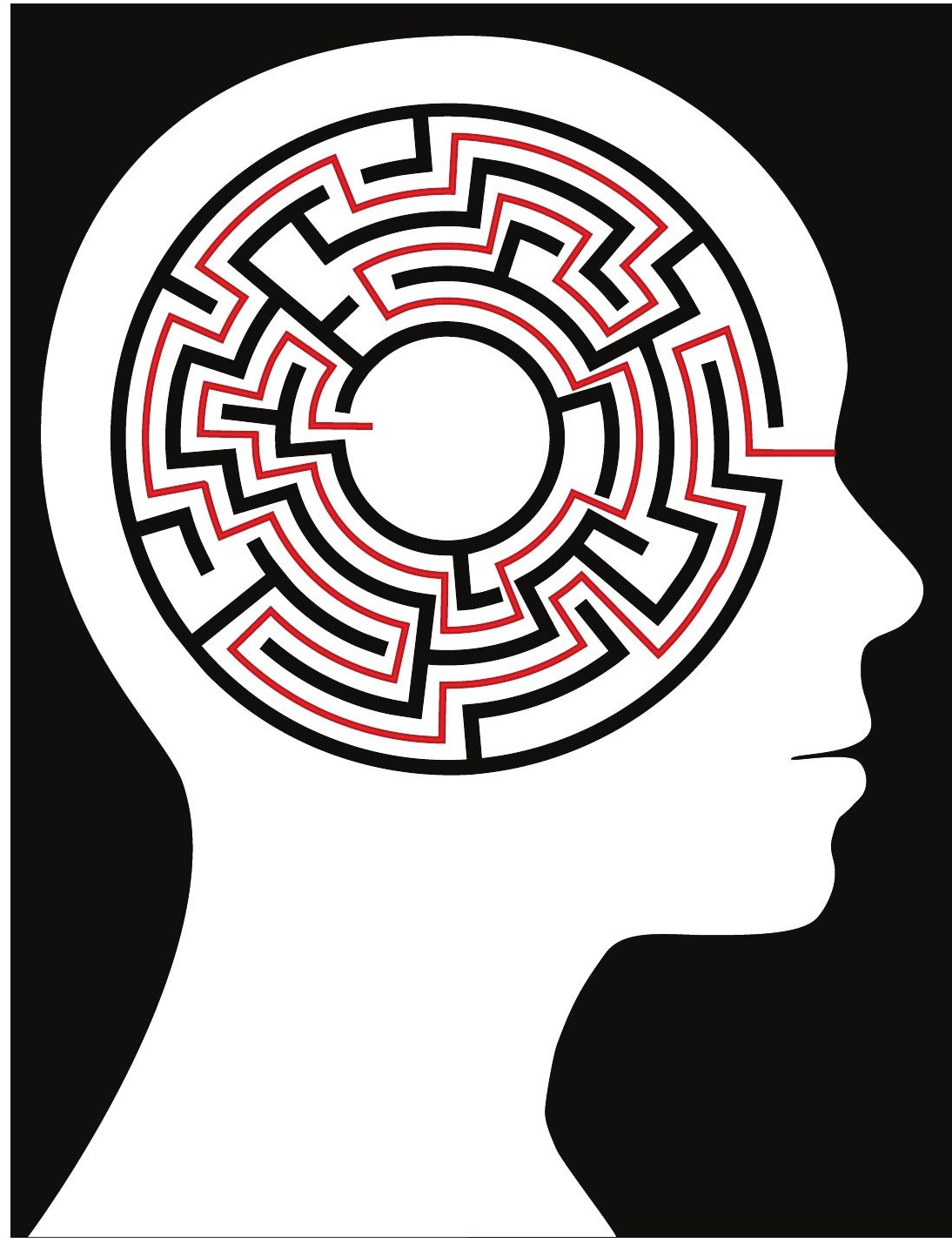

Department of Neurology, University of Rochester, Rochester, NY.

Correspondence to: ralph_jozefowicz@urmc.rochester.edu 


\section{Conversion disorder is defined by neurologic symptoms without evidence of an underlying neurologic disease and generally appears as motor or sensory complaints or seizure-like activity.}

\section{Somatization disorder}

Case report A 34-year-old woman presented for a second opinion concerning multiple sclerosis (MS). She developed low back pain after a work injury at age 26. She then developed paresthesias in her feet that progressed to stocking-glove numbness. She also reported intermittent hand twitches, an episode of right eye pain with blurry vision, and a 10-minute convulsive event during which she was able to visually track her boyfriend but unable to communicate. In addition, she reported vertigo, hoarseness, dysphagia, pale fingers, joint swelling, constipation, nighttime urinary frequency, and that her thighs became stuck in an abducted position following sexual intercourse. Her examination revealed reduced sensation to all modalities in a stocking-glove distribution. Prior workup with extensive laboratory testing, MRI, EEG, and lumbar puncture was unremarkable. Repeat EEG and visual evoked responses were normal.

A disorder characterized by multiple, nonspecific medical complaints has been described since antiquity, when it was termed hysteria due to suspected association with a wandering uterus. ${ }^{4}$ The DSM-IV simplified DSM-III criteria for somatization disorder (or Briquet syndrome), citing recurrent, multiple somatic complaints resulting in medical attention or disability as the key features of the disorder.

SD is observed worldwide, more often in women, with first symptoms appearing by age 25 . Although typically chronic, milder forms remit in approximately $50 \%$ of cases, similar to other medically unexplained symptoms. ${ }^{5}$ Comorbid depression, anxiety, and substance use is common, as are personality disorders and childhood abuse. ${ }^{6}$ Difficulty with prior physicians and a record of repeated workup including exploratory surgery are common. ${ }^{7}$

Somatization may be suspected when the panoply of dysfunctional organ systems is belied by the patient's apparent health. A constellation of pain complaints and gastrointestinal, sexual, and pseudoneurologic symptoms is required for a DSM-IV diagnosis of somatization disorder. ${ }^{3}$ We have found that being provided an exhaustively detailed, self-written history or timeline suggests the diagnosis.

Workup should exclude plausible diagnoses, especially if objective signs are present, but extensive evaluation is best avoided. Functional imaging studies have demonstrated alterations in cortical and limbic networks, which promote disengagement from and suppression of mild or chronic pain, ${ }^{8}$ but these procedures have no role in current diagnostic testing.

Benefit has been demonstrated from psychotherapy, including cognitive behavioral therapy (CBT). ${ }^{9}$ Since permanent remission is rare, management depends on creating a stable relationship with regular, scheduled follow-up (usually best done by the primary care physician), allowing for judicious workup of objectively corroborated problems, while reducing the need for the patient to develop novel symptoms to access care. ${ }^{10}$

In our case, we considered the diagnosis of hypochondriasis, but the widespread complaints including pain, gastrointestinal symptoms, and sexual dysfunction strongly suggest somatization disorder. We offered this diagnosis to the patient after inviting her to discuss possibilities she would not consider, including psychiatric diagnoses. We then presented the DSM-IV 


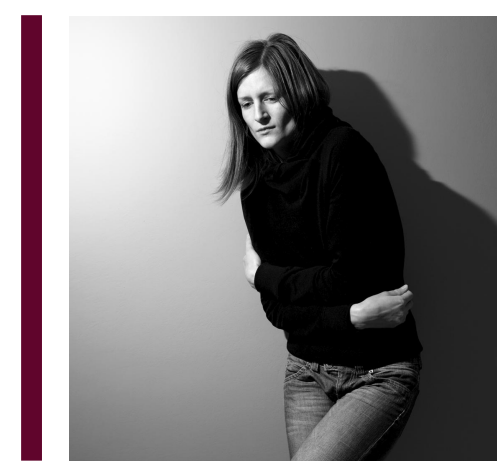

\section{A constellation of pain complaints and gastrointestinal, sexual, and pseudo-neurologic symptoms is required for a DSM-IV diagnosis of somatization disorder.}

criteria to her, which we have previously found to be a successful strategy, since it legitimizes the diagnosis by providing the patient a credible medical reference.

\section{Conversion disorder}

Case report $A$ 45-year-old woman is referred for a second neurologic opinion because of slurred speech. She has seen numerous physicians over the past 6 months for this problem. At one point she was told she "may have myasthenia gravis" and was treated with pyridostigmine, which had no effect. She notes no difficulty swallowing and has maintained a stable weight. Although concerned about her symptoms, she denies anxiety or depression. On examination, her speech has a nasal quality, but the palate elevates normally and the gag reflex is intact. Her ocular motions are full, there is no ptosis, and her facial strength and sensation are normal. Her motor and sensory examinations are normal. She has $2+$ reflexes bilaterally and flexor plantar responses. Her gait is normal. Further questioning reveals that her symptoms developed after an argument with her husband, during which he became verbally abusive. During a double-blind Tensilon test, her speech normalizes immediately following injection of placebo.

Conversion disorder is defined by neurologic symptoms without evidence of an underlying neurologic disease and generally appears as motor or sensory complaints or seizure-like activity. The term was popularized by Freud, who espoused the concept that psychological distress was "converted" into a representative physical ailment. ${ }^{4}$ Conversion is more common in women, and presents throughout the lifespan. ${ }^{3}$

Conversion may be suspected with a history of atypical features of neurologic diseases, or if localization requires an implausible lesion in terms of neuroanatomy. Although included in the $D S M$ criteria, psychological stressors are often not identifiable. Symptoms and signs present mainly in conversion are listed in table 1. Recent data suggest that fears of misdiagnosing neurologic symptoms as unexplained by underlying disease are largely unfounded, with only $0.4 \%$ of patients ultimately found to have an explanatory illness. ${ }^{11}$

In general, a diagnosis of conversion should only be made following a thorough review of the medical record, a detailed history and neurologic examination, and complete MRI of relevant portions of the neuraxis. Electrophysiologic or laboratory testing may also be necessary to rule out specific processes or establish the diagnosis, as with video-EEG monitoring for psychogenic nonelectrical attacks (PNEA). Functional neuroimaging demonstrates increased activity in networks involved in the processing of emotion and affect, ${ }^{8}$ but does not play a role in clinical diagnosis.

We recommend presenting the diagnosis positively to patients by telling them they have an identifiable illness, namely conversion disorder, and by reviewing any symptoms and signs suggestive of conversion as well as the normal objective examination findings and negative test results. To help the patient understand the concept of conversion, we often refer to examples 
Table 1 Common symptoms of conversion, suggestive historical features, and helpful diagnostic maneuvers ${ }^{a}$

\begin{tabular}{|c|c|}
\hline Symptom & $\begin{array}{l}\text { Historical features or examination findings } \\
\text { that suggest conversion }\end{array}$ \\
\hline \multirow[t]{4}{*}{ Unresponsive } & Controls descent of dropped hand \\
\hline & Eyes held shut \\
\hline & Responds to painful stimulus \\
\hline & Sleeps \\
\hline \multirow[t]{11}{*}{ Convulsions } & Asynchronous, semi/purposeful movements \\
\hline & Eyes held shut \\
\hline & Intraictal response to touch \\
\hline & Opisthotonus \\
\hline & Pelvic thrusting \\
\hline & Postictal weeping \\
\hline & Prolonged attack \\
\hline & Rapid recovery \\
\hline & Side-side head shaking \\
\hline & Stuttering \\
\hline & $\begin{array}{l}\text { "Teddy bear" sign (patient is accompanied by an age-inappropriate } \\
\text { stuffed animal) }\end{array}$ \\
\hline \multirow[t]{5}{*}{ Weakness } & Collapsing weakness \\
\hline & Downward drift of outstretched arm without pronation \\
\hline & Dragging monoplegic gait ${ }^{37}$ \\
\hline & $\begin{array}{l}\text { Hoover sign (weakness of hip extension improves with contralateral } \\
\text { hip flexion) }\end{array}$ \\
\hline & Inconsistency across postures or time \\
\hline \multicolumn{2}{|c|}{ Abnormal movement } \\
\hline \multirow[t]{3}{*}{ Dystonia } & Absence of sensory trick or overflow dystonia \\
\hline & Clenched fist \\
\hline & Inverted ankle \\
\hline \multirow[t]{4}{*}{ Tremor } & Distractibility \\
\hline & $\begin{array}{l}\text { Entrainment (affected hand mimics rhythmic movements of normal } \\
\text { hand) }\end{array}$ \\
\hline & Variability \\
\hline & Worse with loading or resistance \\
\hline \multirow[t]{4}{*}{ Sensory } & Acral distribution, especially with central facial involvement \\
\hline & Midline splitting \\
\hline & Splitting of vibration (sternum, frontal bone) \\
\hline & Tubular or spiral visual fields \\
\hline
\end{tabular}

a It should be emphasized that nothing listed is an absolute indicator of conversion or other somatoform disorders, but in the proper constellation, may assist in making the diagnosis. Of note, the classic description of an indifferent, cheerful mien (la belle indifférence), may not be useful, as it is often seen with medical illness. ${ }^{31}$ The authors wish to credit reviews by Aybek et al., ${ }^{32}$ Hawkes, $^{33}$ Shill and Gerber, ${ }^{34}$ and Stone and Sharpe. ${ }^{35}$ 
of other common somatoform symptoms, such as increased gastric acid secretion due to stress, irritable bowel syndrome, stress as a trigger for migraine, and stomach butterflies with public speaking. It is important to reiterate that the patient is not manufacturing his or her symptoms, and to affirm that help will be provided.

CBT has been found to be effective in paroxysmal nonepileptic attacks. ${ }^{12,13}$ We have occasionally suggested that patients consider transition to a new therapist if their current provider is not experienced with treating conversion. Selective serotonin reuptake inhibitors (SSRI) may be beneficial. ${ }^{14}$ The goal of treatment is both to alleviate symptoms and reduce unnecessary medical care. Clear communication may reduce redundant testing by an unfamiliar provider.

Our case illustrates how conversion may approximate a neurologic disorder, but ultimately lacks expected examination findings or associated deficits. The reversal of symptoms with injection of placebo established the diagnosis. The presence of clear psychological trauma is helpful, but not required to make the diagnosis.

\section{Pain disorder}

Case report $A$ 44-year-old truck driver is referred from a pain treatment center for a second opinion because of "total body pain" that he describes as "burning, sharp, stabbing, tingling, and hot." His symptoms began 5 years ago when he dislocated his left shoulder while rearranging a tarp on his truck, following which he developed persistent, sharp left shoulder blade pain with radiation into the arm. Physical therapy, splinting, oral analgesics, muscle relaxants, stellate ganglion block, spinal epidural injections, dorsal column stimulator, and subacromial decompression surgery were ineffective in treating his symptoms. The pain subsequently spread into the jaw, tongue, right arm, and both lower extremities. He has seen numerous specialists, including orthopedic surgeons, neurologists, psychiatrists, and a pain specialist, all without substantive relief, and was told that he had "atypical total body reflex sympathetic dystrophy" by a pain specialist. On examination, he grimaces frequently when not being engaged. He is exquisitely tender over the entire body, but lacks any signs of complex regional pain syndrome. He has diffuse give-away weakness in all limbs. Workup, including MRI of the brain and cervical spine, EMG, and nerve conduction studies, is normal.

Pain disorder is characterized by pain disproportionate to any underlying injury or illness, to the point of significant functional impairment. ${ }^{3}$ Prevalence in the community is as high as $8.3 \%$, and neurologists will not be surprised that headache represents the most common pain complaint. ${ }^{16}$ However, overlap with other somatoform disorders continues to foster debate on the validity of a pure pain somatoform disorder. ${ }^{15,16}$ The biological underpinnings of pain disorder may involve emotional dysregulation via modulation of limbic-cortical networks involving the amygdala and anterior cingulate gyrus. ${ }^{17,18}$

Although head and musculoskeletal pain may present more often in women, pain disorder presents in all ages and demographics. ${ }^{3}$ Comorbid psychiatric diagnoses are common, especially anxiety, depression, and personality disorders. ${ }^{19}$

Diagnostic tip-offs include poorly localized, intransigent, vaguely described pain that lacks aggravating or relieving factors, ${ }^{20}$ showing little or no response to a variety of reasonable treatment options. Testing is directed at excluding reasonable alternatives. Treatment options include medications, particularly tricyclic antidepressants and SSRIs, ${ }^{21,22}$ and psychotherapy. ${ }^{23}$ Nonpharmacologic treatment strategies (e.g., massage, acupuncture) may also be beneficial.

Our patient initially presented with symptomatic pain due to a shoulder injury. But then, rather than resolving, his pain persisted and generalized to the point of causing total disability. Extensive evaluation by numerous specialists had been unrevealing and treatment has been ineffective. The diffuse nature of his pain and the lack of benefit from a variety of modalities suggest pain disorder as the diagnosis. Judicious use of non-narcotic pharmacotherapy and 
Table 2 Central principles for the diagnosis and treatment of somatoform disorders ${ }^{a}$

\section{Basic tenets of care}

Perform a complete physical examination initially

Establish a long-term relationship

\section{Be aware of countertransference}

\section{Do not rush to determine or confront the psychological basis of symptoms}

\section{Key elements of understanding and means of implementation}

Examination helps rule out organic illness

"Laying on of hands" indicates concern

Periodic reassessment reduces diagnostic uncertainty, detects new problems, and continues emotional support

Schedule regular follow-up visits

Allow impromptu visits for reassurance

Set limits if patient abuses the physician's time

Realize that symptoms are an emotional communication rather than a sign of illness

\section{Respect the adaptive value of the symptoms}

Understand that the patient wants palliation (continued care) and not cure (termination of care)

A physician's emotional reactions to the patient may be influenced by the physician's own unconscious conflict

Awareness of the psychological cause is not necessary for improvement

Resistance to psychiatric referral is common

Present referral as an opportunity to learn alternative adaptive strategies

Avoid unnecessary laboratory testing

Avoid psychotropics and prescription analgesics

Time is an extremely effective test

Follow-up over several weeks may result in resolution of symptoms

a These concepts illustrate one approach to dealing with a variety of somatoform presentations. Although this is by no means the only viable set of guiding principles, we have found it effective and relatively portable across the spectrum of somatoform disorders.

psychotherapy both play a role in treatment; narcotics should be avoided due to their lack of long-term efficacy and high potential for addiction.

\section{Factitious disorder}

Case report While riding a bus in upstate New York, a 68-year-old man complained of severe chest pain, nausea, and dyspnea, followed by left body and right face paresthesias, left-sided weakness, and word-finding difficulty. He was brought to a nearby major medical center. Shortly following arrival in the emergency department, he was reported to have a generalized convulsion. On examination, he was lethargic, disoriented, dysarthric, and was making frequent paraphasic errors. He had difficulty looking right and displayed a left facial droop. He was unable to move the left side against gravity. He reported reduced sensation to pain and temperature on the right face and left body. Chest $x$-ray and ECG were normal. CT of the head revealed right cerebellar hypodensities consistent with prior infarction. Stroke of the right medulla and a separate cortical infarct were suspected, but EEG, echocardiogram, and MRI of the brain were unrevealing. The following day he was noted by the nursing staff to have another convulsion, after which the neurologic deficits he had at presentation were no longer evident. Several hours later he complained of inconsistent right visual field impairment. When a cerebral angiogram was recommended to rule out vasculitis, he became agitated and combative, demanding to leave the hospital and threatening litigation. Collateral information from an out-of-state landlord revealed thousands of dollars in unpaid ambulance fees, and frequent, similar queries from physicians throughout the country. 
When confronted with this history, the patient suddenly became scared and said "Doc, I promise, I won't do hospitals anymore."

Recurrent, intentional falsification is the primary feature of FD, which must lack the clear secondary gain of malingering. Symptoms and signs are feigned or self-inflicted to mimic one or more medical conditions, e.g., contaminating an IV site with fecal matter or injecting exogenous insulin to produce symptomatic hypoglycemia.

FD begins in adulthood and may persist lifelong. ${ }^{3}$ Though most common in young women, it may be more severe in men, who are more likely to show peregrinatory behavior (Munchausen syndrome). ${ }^{24,25}$ Some patients may incorporate undisclosed preexisting conditions (such as the cerebellar infarcts in our patient). Personality disorders, especially borderline, are associated with FD, as is substance abuse, childhood abuse, and exposure to or employment in medical settings. ${ }^{25,26}$ Prevalence is difficult to establish, in part because a patient may present to multiple facilities under different names, but is reported as high as $1.3 \%$ in hospital clinics. ${ }^{3,26,27}$

Neurologic complaints include pain, seizure, or dizziness, although other dysfunctions may occur. Red flags include a dramatic history, vague or variable in its detail, delivered with a highly medicalized vocabulary while glossing over the origin of scars from invasive procedures. Attempts to corroborate the history reveal incessant confabulation even on irrelevant details. Peregrination may be more frequent in those with primarily neurologic complaints. ${ }^{24,28} \mathrm{New}$ symptoms often emerge as previous possibilities are eliminated. The disorder may also present "by proxy," with the affected individual inducing symptoms in another person.

Definitive diagnosis requires observation of deliberate production of symptoms (or remission of deficits when the patient believes himself or herself to be unobserved), but the presence of inexplicable laboratory results, inconsistent history and examination findings, and collateral information create a high index of suspicion. ${ }^{25}$ There are few data to suggest whether or not to confront patients with the diagnosis, as the reaction ranges from denial to threats of litigation or outright flight. ${ }^{29}$ Similarly, no therapeutic regimen shows clear advantage. The clinician may have to navigate these decisions while dealing with negative emotional reactions. Diagnosis must be sought, as there is a real risk of serious self-inflicted injury or death, ${ }^{30}$ in addition to the risks of repeated unnecessary evaluation and treatment.

Our case highlights the migratory nature of the factitious patient with Munchausen syndrome. The use of existent physical signs and confabulated symptoms is characteristic, as is development of new symptoms when the initial concern has been ruled out. The abrupt departure is also characteristic, and often prevents engagement over the diagnosis.

\section{CONCLUSIONS}

Effective treatment of patients with somatoform disorders is difficult, time consuming, and requires patience and understanding. Organic medical conditions must be ruled out, followed by psychiatric conditions, before a positive diagnosis of a somatoform disorder is confirmed and a strategy for further care agreed upon. A general framework to approach these disorders, which we have found beneficial, is presented in table 2.

Ongoing debate continues on how to define and categorize the disorders presented above, challenging the validity of the DSM categorization and often the concept of mind-body dualism as a helpful construct. Although the formal categorization may change with upcoming revisions to the DSM and International Classification of Diseases, patients with these problems will continue to present to neurologists with a variety of complaints. Familiarity with the themes of these disorders will enable the clinician to pursue a positive diagnosis, while using judicious testing to rule out underlying medical problems. The benefit of making a diagnosis may vary given the limited treatment options for some disorders and the chronic course of others, but at the very least it will lay the groundwork for subsequent visits and the appropriate evaluation of new concerns. 


\section{REFERENCES}

1. Mace C, Trimble M. 'Hysteria,' functional' or 'psychogenic'? A survey of British neurologists' preferences. J R Soc Med 1991;84:471-475.

2. Nimnuan C, Hotopf M, Wessely S. Medically unexplained symptoms: an epidemiological study in seven specialties. J Psychosom Res 2001;51:361-367.

3. American Psychiatric Association. Diagnostic and Statistical Manual of Mental Disorders: DSM-IV, Text Revision. Washington, DC: American Psychiatric Association; 2000.

4. Noyes RJ, Stuart S, Watson D. A reconceptualization of the somatoform disorders. Psychosomatics 2008;49:14-22.

5. Gureje O, Simon G. The natural history of somatization in primary care. Psychol Med 1999;29:669676.

6. Spitzer C, Barnow S, Gau K, Freyberger HJ, Grabe HJ. Childhood maltreatment in patients with somatization disorder. Aust NZ J Psychiatry 2008;42:335-341.

7. Monson R, Smith GJ. Current concepts in psychiatry: somatization disorder in primary care. N Engl J Med 1983;308:1464-1465.

8. Garcia-Campayo J, Fayed N, Serrano-Blanco A, Roca M. Brain dysfunction behind functional symptoms: neuroimaging and somatoform, conversive, and dissociative disorders. Curr Opin Psychiatry 2009;22:224-231.

9. Allen L, Woolfolk R. Cognitive behavioral therapy for somatoform disorders. Psychiatr Clin North Am 2010;33:579-593.

10. Servan-Schreiber D, Kolb R, Tabas G. The somatizing patient. Prim Care 1999;26:225-242.

11. Stone J, Carson A, Duncan R, et al. Symptoms 'unexplained by organic disease' in 1144 new neurology out-patients: how often does the diagnosis change at follow-up? Brain 2009;132:2878-2888.

12. Goldstein LH, Deate AC, Mitchell-O’Malley SJ, Toone BK, Mellers JD. Cognitive-behavioral therapy for psychogenic nonepileptic seizures: a pilot RCT. Neurology 2010;74:1986-1994.

13. LaFrance WJ, Miller IW, Ryan CE, et al. Cognitive behavioral therapy for psychogenic nonepileptic seizures. Epilepsy Behav 2009;14:591-596.

14. LaFrance WJ, Keitner GI, Papandonatos GD, et al. Pilot pharmacologic randomized controlled trial for psychogenic nonepileptic seizures. Neurology 2010;75:1166-1173.

15. Fröhlich C, Jacobi F, Wittchen H. DSM-IV pain disorder in the general population: an exploration of the structure and threshold of medically unexplained pain symptoms. Eur Arch Psychiatry Clin Neurosci 2006;256:187-196.

16. Nordin H, Eisemann M, Richter J. The accuracy of the DSM-IV pain disorder and the ICD-10 persistent somatoform pain disorder in chronic pain patients. J Clin Psychol Med Settings 2006;13: 303-310.

17. Gundel H, Valet M, Sorg C, et al. Altered cerebral response to noxious heat stimulation in patients with somatoform pain disorder. Pain 2008;137:413-421.

18. Klug S, Anderer P, Saletu-Zyhlarz G, et al. Dysfunctional pain modulation in somatoform pain disorder patients. Eur Arch Psychiatry Clin Neurosci 2011;261:267-75.

19. Dohrenwend A, Lyon Skillings J. Diagnosis-specific management of somatoform disorders: moving beyond "vague complaints of pain." J Pain 2009;10:1128-1137.

20. Adler R, Zamboni P, Hofer T, et al. How not to miss a somatic needle in the haystack of chronic pain. J Psychosom Res 1997;42:499-505.

21. Luo Y, Zhang MY, Wu WY, Li CB, Lu Z, Li QW. A randomized double-blind clinical trial on analgesic efficacy of fluoxetine for persistent somatoform pain disorder. Prog Neuropsychopharmacol Biol Psychiatry 2009;33:1522-1525.

22. O'Malley P, Jackson JL, Santoro J, Tomkins G, Balden E, Kroenke K. Antidepressant therapy for unexplained symptoms and symptom syndromes. J Fam Pract 1999;48:980-990.

23. Nickel R, Ademmer K, Egle U. Manualized psychodynamic-interactional group therapy for the treatment of somatoform pain disorders. Bull Menninger Clin 2010;74:219-237.

24. Asher R, Long M. Munchhausen's syndrome. Lancet 1951;1:339-341.

25. Krahn L, Li H, O'Connor M. Patients who strive to be ill: factitious disorder with physical symptoms. Am J Psychiatry 2003;160:1163-1168.

26. Sutherland A, Rodin G. Factitious disorders in a general hospital setting: clinical features and a review of the literature. Psychosomatics 1990;31:392-399.

27. Fliege H, Grimm A, Echkardt-Henn A, Gieler U, Martin K, Klapp BF. Frequency of ICD-10 factitious disorder: survey of senior hospital consultants and physicians in private practice. Psychosomatics 2007; 48:60-64.

28. Kanaan R, Wessely S. Factitious disorders in neurology: an analysis of reported cases. Psychosomatics 2010;51:47-54. 
29. Feldman M, Feldman J. Tangled in the web: countertransference in the therapy of factitious disorders. Int J Psychiatry Med 1995;25:389-399.

30. Eisendrath S, McNiel D. Factitious physical disorders, litigation, and mortality. Psychosomatics 2004; 45:350-353.

31. Stone J, Smyth R, Carson A, Warlow C, Sharpe M. La belle indifférence in conversion symptoms and hysteria: systematic review. Br J Psychiatry 2006;188:204-209.

32. Aybek S, Kanaan R, David A. The neuropsychiatry of conversion disorder. Curr Opin Psychiatry 2008;21:275-280.

33. Hawkes C. Diagnosis of functional neurological disease. Br J Hosp Med 1997;57:373-377.

34. Shill H, Gerber P. Evaluation of clinical diagnostic criteria for psychogenic movement disorders. Mov Disord 2006;21:1163-1168.

35. Stone J, Sharpe M. Functional symptoms in neurology: case studies. Neurol Clin 2006;24:385-403.

36. Burneo J, Martin R, Powell T, et al. Teddy bears: an observational finding in patients with nonepileptic events. Neurology 2003;61:714-715.

37. Stone J, Zeman A, Sharpe M. Functional weakness and sensory disturbance. J Neurol Neurosurg Psychiatry 2002;73:241-245.

38. Todd R. Clinical Lectures on Paralyses, Diseases of the Brain, and Other Affections of the Nervous System. London: Churchill; 1854.

39. Barsky AJ. A 37-year old man with multiple somatic complaints (Clinical Crossroads). JAMA 1997; 278:673-679.

40. Drossmasn DA. The problem patient. Ann Intern Med 1978;88:366-372.

\section{DISCLOSURES}

The authors report no disclosures.

\section{Related articles from other AAN physician and patient resources}

Neurology ${ }^{\circledR} \quad$ www.neurology.org

Guided self-help for functional (psychogenic) symptoms: A randomized controlled efficacy trial August 9, 2011;77:564-572.

Cohort study on somatoform disorders in Parkinson disease and dementia with Lewy bodies May 18, 2010;74:1598-1606.

The involuntary nature of conversion disorder January 19, 2010;74:223-228.

\section{Continuum: Lifelong Learning in Neurology ${ }^{\circledR} \quad$ www.aan.com/continuum}

\section{Somatization - An Overview for Neurologists}

October 2006;12:87-99.

\section{Neurology Today ${ }^{\circledR} \quad$ - www.neurotodayonline.com}

Cognitive Therapy Helpful in Reducing Pseudoseizures, First Randomized Trial Finds June 17, 2010;10:5,8,9. 